\title{
Femoral component failure in the Oxford unicompartmental knee arthroplasty: a case report
}

\author{
Kirsten DS Argelo', Mick A Burger ${ }^{1}$, Marco JM Hoozemans ${ }^{1,2}$ and Olivier PP Temmerman ${ }^{1,3^{*}}$
}

\begin{abstract}
Introduction: The present case report describes a patient who presented with an early complication after a unicompartmental knee arthroplasty. It is not the first case in this subject but the unique aspect of this case report rests on the timing in which the failure occurred.

Case presentation: A 64-year-old Caucasian man received a medial unicompartmental knee arthroplasty (Oxford ${ }^{\varpi}$ Partial Knee) due to isolated anteromedial osteoarthritis of his right knee. His initial recovery was good, however, after 3 months he presented with acute pain and a locked knee. Radiographs showed a complete loosening and migration of the femoral component. During revision surgery no clear explanation was found for failure of the femoral component.

Conclusions: The most likely explanation for loosening is the combination of peak stresses on the posterior facet of the femoral components of a unicompartmental knee arthroplasty in a patient in a cross-legged knee position causing bone-cement or cement-implant interface failure. Further research is necessary in prosthetic designs and applications of the unicompartmental knee arthroplasty to determine the origin of this early complication.
\end{abstract}

Keywords: Complication, Knee arthroplasty, Unicompartmental knee arthroplasty

\section{Introduction}

In orthopaedic patients that present with a painful knee, osteoarthritis is a commonly found diagnosis. The incidence of osteoarthritis of the knee (OAK) in The Netherlands in 2007 was estimated at 1.6:1000 for men and 3.1:1000 for women [1]. End-stage OAK generally results in unicompartmental or total knee arthroplasty. It is expected that the ageing population will increase the need for unicompartmental and total knee arthroplasties [1]. Approximately $10 \%$ of the patients that undergo a knee arthroplasty are treated with a unicompartmental knee arthroplasty (UKA) [2]. Specific inclusion criteria are used for UKA, in particular, isolated osteoarthritis in the anteromedial tibiofemoral compartment and an intact anterior cruciate ligament.

For this specific patient population UKA is associated with less invasive surgery and a faster postoperative

\footnotetext{
* Correspondence: o.p.p.temmerman@mca.nl

'CORAL - Centre for Orthopaedic Research Alkmaar, Department of Orthopaedic Surgery, Medical Center Alkmaar, P.O Box 501, 1800 AM Alkmaar, The Netherlands

${ }^{3}$ Department of Orthopaedic Surgery, Medical Center Alkmaar, P.O Box 501, 1800 AM Alkmaar, The Netherlands

Full list of author information is available at the end of the article
}

recovery as compared to total knee arthroplasty [2]. The 10-year survival rate of UKA is reported to be over $90 \%$ [3]. However, the absolute number of complications and revisions is expected to increase in accordance with the expected increasing number of UKA that are going to be performed. The present case report describes a Caucasian patient who presented at our orthopaedic outpatient department with an early complication after a UKA.

\section{Case presentation}

A 64-year-old Caucasian man visited our out-patient department with symptomatic anteromedial osteoarthritis of his right knee. A physical examination revealed a full range of motion and stable collateral and cruciate ligaments. Plain radiography showed an anteromedial osteoarthritis grade III [4] (Figure 1). After an initial conservative approach, which included a 1-year period of physiotherapy, non-steroidal anti-inflammatory drugs and three hyaluronic acid injections, he was planned for a UKA (Oxford ${ }^{\circ}$ Partial Knee).

Preoperatively, a medium-size femoral component was templated. The surgery was performed by a specialist arthroplasty surgeon. During surgery, a femur of medium 


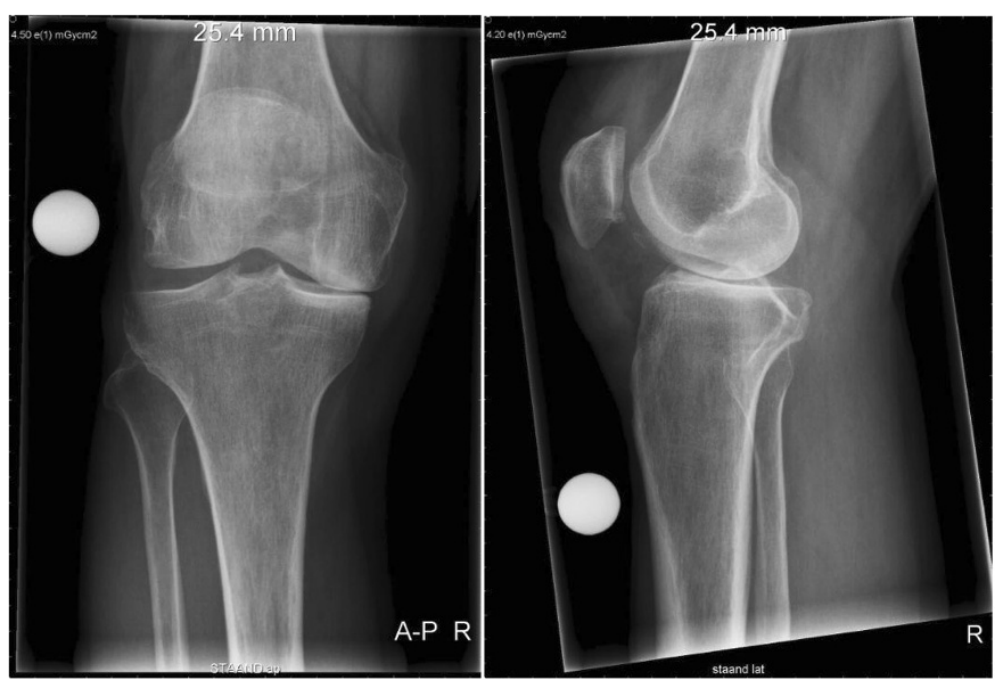

Figure 1 Preoperative anterior-posterior and lateral X-rays showing anteromedial osteoarthritis of the right knee.

size, a tibia size $C$ and an insert size 3 were placed and no complications were experienced. The preoperative diagnosis of anteromedial osteoarthritis was confirmed and the anterior cruciate ligament was found to be stable. The time of blood void was 63 minutes and the bone quality of both tibia and femur was assessed as good. Pulsed lavage was applied to increased femoral cement penetration in combination with the application of retention holes to further enhance cement fixation. Range of motion of the knee after wound closure was 120 degrees of flexion and full extension with stable collateral and cruciate ligaments. The postoperative $\mathrm{X}$-rays revealed no abnormalities and proper positioning of the UKA (Figure 2). The postoperative checks at the out-patient department were planned 6 weeks and 3 months postoperatively. At both appointments the patient showed a good clinical recovery and his range of motion observed at 3 months was 120/0/0.

Two weeks after the last check he presented at the outpatient department with extreme pain and a locked knee. There was no trauma; however, he reported an acute pain
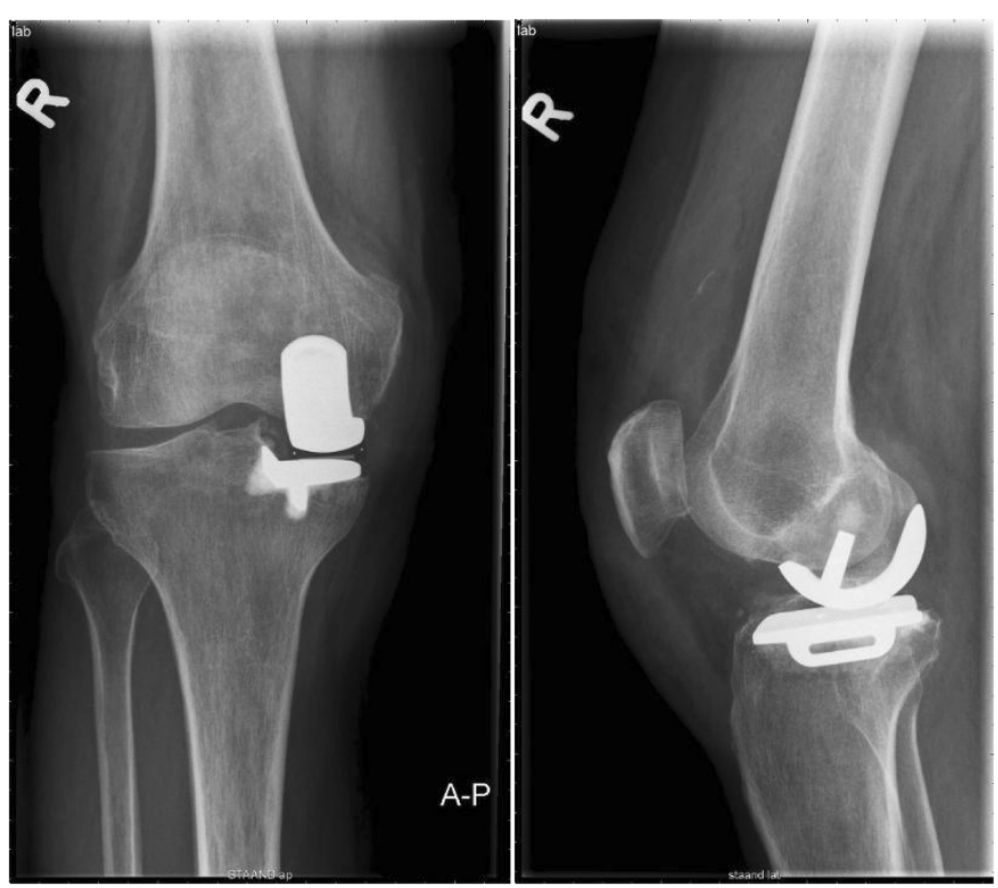

Figure 2 Postoperative anterior-posterior and lateral X-rays directly after the insertion of an Oxford unicompartmental knee prosthesis. 
after sitting in a cross-legged position. A physical examination showed that he was in pain with a locked knee in 90/90/0. An X-ray showed a fully migrated and loosened femoral component (Figure 3).

During revision surgery, performed by the same surgeon, a crack in the cement of the femoral component was observed (Figures 4, 5 and 6). No other signs were found that could explain failure of the prosthesis. Cultures and biopsies of the medial femur condyle were taken for further examination; however, bacterial infection or osteonecrosis was not found. The tibial component was found to be solid. During revision surgery, a total knee arthroplasty (Genesis II Total Knee System, Smith \& Nephew $^{\circ}$, Memphis, USA) with a femur size 7 posterior stabilized component was placed in combination with a tibia size 6, an insert size 15 and patella size 26. During surgery no complications were experienced. His range of motion after wound closure was 120 degrees of flexion

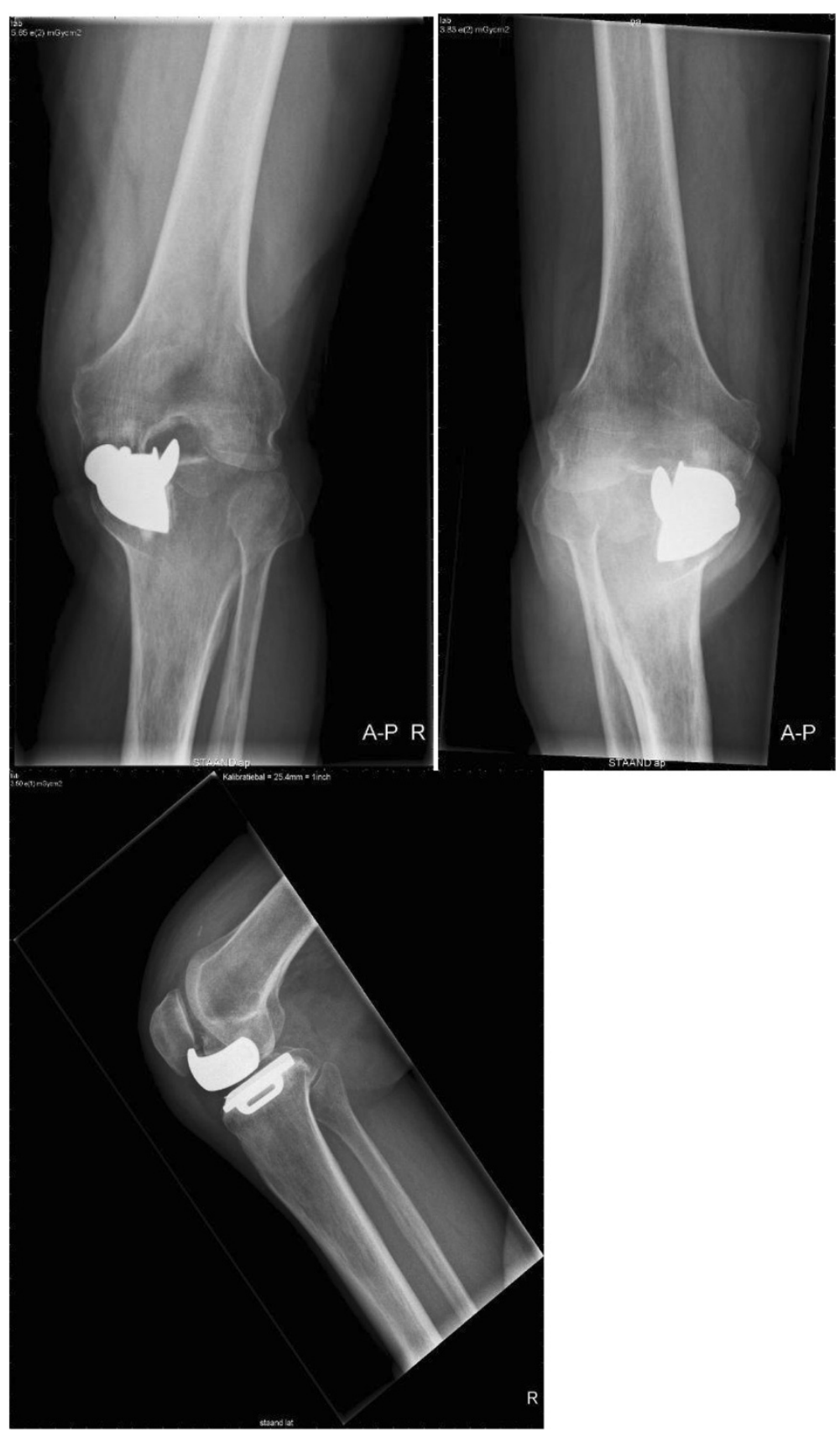

Figure 3 Anterior-posterior and lateral X-rays at 2 weeks after the 3-month postoperative check-up with the patient reporting extreme pain and a locked knee. 


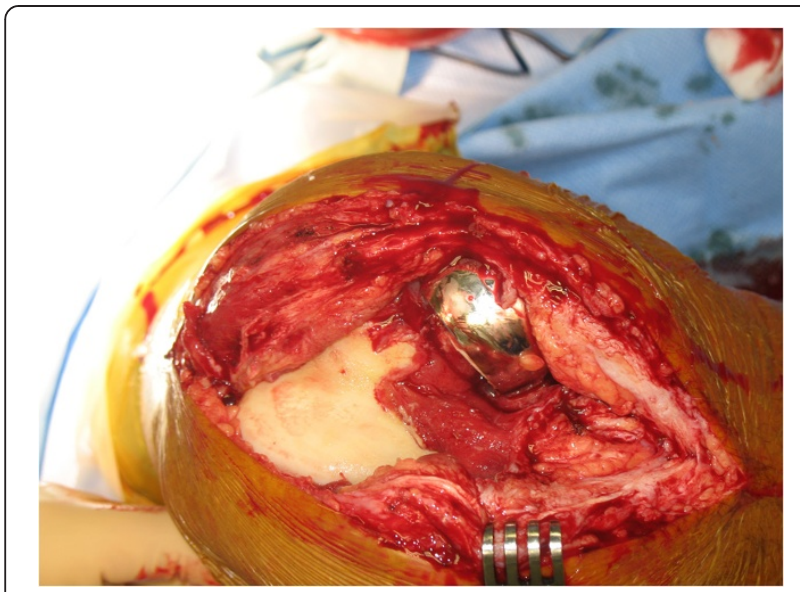

Figure 4 Photograph of the dislocated femoral component during surgery.

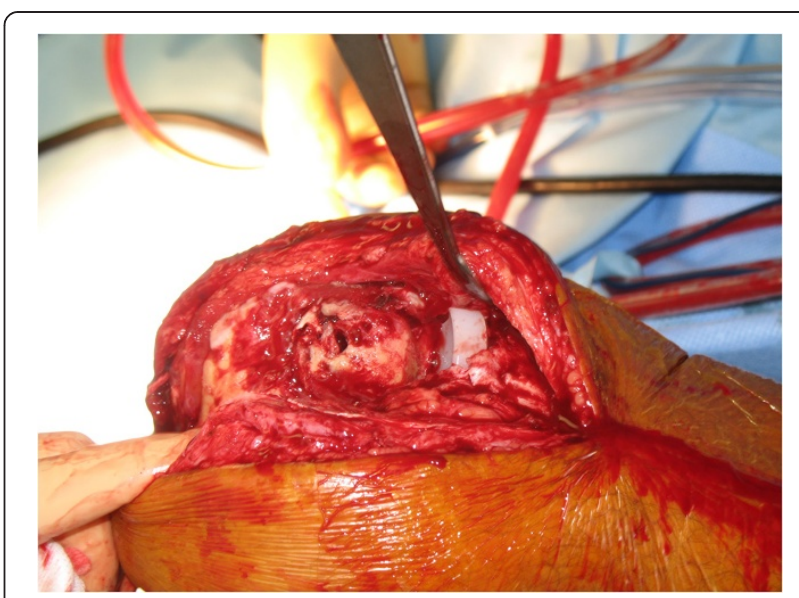

Figure 6 Photograph of the femoral condyle after removal of the femoral component of the Oxford unicompartmental knee prosthesis. and full extension with stable collateral ligaments. The postoperative $\mathrm{X}$-rays revealed no abnormalities and a proper positioning of the total knee prosthesis.

His recovery after revision surgery was without complications. During the last appointment at the out-patient department, 1 year after revision surgery, he showed a good clinical recovery and the range of motion observed was $120 / 0 / 0$.

\section{Discussion}

UKA is a well-established and proven method to reduce symptoms and disabilities associated with (end-stage) anteromedial tibiofemoral osteoarthritis. UKA is less invasive compared to total knee arthroplasty and postoperatively associated with less pain, less blood loss, better proprioception and faster recovery [2,5-7]. Complications of UKA

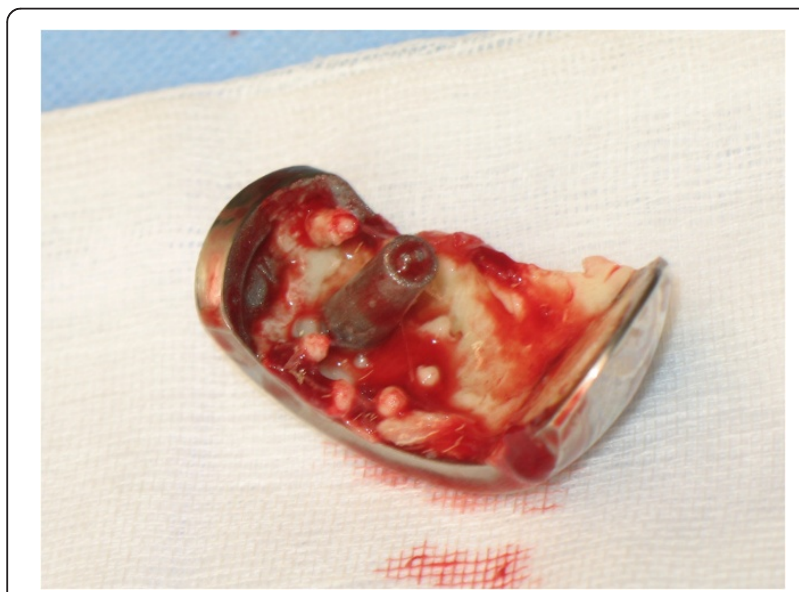

Figure 5 Photograph of the removed femoral component, with a crack in the cement. reported in the literature are aseptic loosening, luxation of the insert, persisting pain postoperatively, persisting stiffness postoperatively, and deep infection [3]. Deep infection is a commonly described complication in arthroplasty [8]. However, in our case, infection was not found in cultures and biopsies. Similarly, other causes and treatments of persistent postoperative anteromedial pain in UKA have been described [2,9-12]. Concerning failures of the UKA, a loosened tibia component is more often reported [13,14]. Technical failure, such as making the sagittal cut at the preparation of the tibia too deep, is most often considered the cause of this type of failure.

Although long-term follow-up studies of the Oxford unicompartmental knee prosthesis have shown excellent outcomes, loosening of the femoral component is the second most common cause of revision and the incidence ranges from zero to $2.1 \%$ [15]. However, failure of the femoral component in the early postoperative phase is rarely reported in the literature [16]. Monk et al. [15] described a technique to detect loosening using lateral radiographs in extension and flexion. If gaps are present between the component and cement on one radiograph and not on the other, the component is considered loose by the authors. We did not acquire these radiographs at the postoperative checks and may therefore have missed loosening of the femoral component.

Another cause of loosening may be smoking. Meldrum et al. [17] calculated a 4.5 times greater risk of implant loosening in cigarette smokers. Cigarette smoking has been shown to interfere with bone metabolism, revascularization and bone formation. Our patient smoked for several years 15 to 20 cigarettes a day. However, it is expected that this type of aseptic loosening is seen in a 
more mid- to long-term follow up and, therefore, is less likely to be an explanation in our case.

Kim et al. describe a possible trauma mechanism, which could explain the failure of the femoral component in the present case [18]. They describe a trauma case with an axial vector action in combination with varus and/or valgus stress, which might have caused a fracture of the medial condyle of the femur. Although there was a suspicion for varus stress in our case, deep flexion was observed instead of axial vector action. Therefore, it is unlikely that the failure of the femoral component observed in the present case is caused by axial vector action.

With regard to the operative technique, a pitfall may lie in the posterior femoral saw cut which can be located too far anteriorly. As a result, the posterior part of the femoral component may be inadequately supported, expressed by a wedge sign, which results in an insufficient prosthesiscement interface $[9,15]$.

Clarius et al. concluded, after a cadaver study in 24 subjects, that the posterior plane facet is proven to be the weak point of the femoral interface because of incompleteness of the cement mantle [9]. In a more recent study Seeger et al. further examined the effects of cementing techniques on implant failure in patients with a UKA (Oxford ${ }^{\circ}$ Partial Knee) [19]. The authors found evidence that the use of pulse lavage decreased the risk of thermal osseous damage and improved cement penetration.

\section{Conclusions}

On reflection, in our case, the cross-legged position may have exerted shear stresses on the posterior plane facet. This, together with an oppressive force on the posterior facet of the femoral component may explain the loosening mechanism in our patient. The visible crack in the cement mantle found during revision surgery may confirm this hypothesis. However, since we have not been able to detect errors in surgical techniques and did apply the current concepts in cementing techniques with regard to preparation and application, the authors feel that future studies should further focus on application and implant design of the UKA.

\section{Consent}

Written informed consent was obtained from the patient for publication of this case report and any accompanying images. A copy of the written consent is available for review by the Editor-in-Chief of this journal.

\section{Abbreviations}

OAK: Osteoarthritis of the knee; UKA: Unicompartmental knee arthroplasty (Oxford ${ }^{\oplus}$ Partial Knee).

\section{Competing interests}

The authors declare that they have no competing interest.

\section{Authors' contributions}

All authors have made substantial contributions to the conception and design of the study and manuscript. All authors read and approved the final manuscript.

\section{Author details}

${ }^{1}$ CORAL - Centre for Orthopaedic Research Alkmaar, Department of Orthopaedic Surgery, Medical Center Alkmaar, P.O Box 501, 1800 AM Alkmaar, The Netherlands. ${ }^{2}$ Research Institute MOVE, Faculty of Human Movement Sciences, VU University Amsterdam, Amsterdam, The Netherlands. ${ }^{3}$ Department of Orthopaedic Surgery, Medical Center Alkmaar, P.O Box 501, 1800 AM Alkmaar, The Netherlands.

Received: 30 April 2014 Accepted: 6 October 2014

Published: 11 December 2014

\section{References}

1. Poos MJJC, Gommer AM, Uiters E, Zantinge EM: Hoe vaak komt artrose voor en hoeveel mensen sterven eraan? [http://www.nationaalkompas.nl/ gezondheid-en-ziekte/ziekten-en-aandoeningen/bewegingsstelsel-enbindweefsel/artrose/omvang/]

2. Heller S, Fenichel I, Salai M, Luria T, Velkes S: The Oxford unicompartmental knee prosthesis for the treatment of medial compartment knee disease: 2 to 5 year follow-up. Isr Med Assoc J 2009, 11(5):266-268.

3. Mercier N, Wimsey S, Saragaglia D: Long-term clinical results of the Oxford medial unicompartmental knee arthroplasty. Int Orthop 2010, 34(8):1137-1143.

4. Kellgren JH, Lawrence JS: Radiological assessment of osteo-arthrosis. Ann Rheum Dis 1957, 16(4):494-502

5. Robertsson O, Bizjajeva S, Fenstad AM, Furnes O, Lidgren L, Mehnert F, Odgaard A, Pedersen AB, Havelin LI: Knee arthroplasty in Denmark, Norway and Sweden. Acta Orthop 2010, 81(1):82-89.

6. Deshmukh RV, Scott RD: Unicompartmental knee arthroplasty: long-term results. Clin Orthop Relat Res 2001, 392:272-278.

7. Murray DW: Mobile bearing unicompartmental knee replacement. Orthopedics 2005, 28(9):985-987.

8. Willis-Owen CA, Konyves A, Martin DK: Factors affecting the incidence of infection in hip and knee replacement: an analysis of 5277 cases. J Bone Joint Surg (Br) 2010, 92(8):1128-1133.

9. Clarius M, Mohr G, Jaeger S, Seeger JB, Bitsch RG: Femoral fixation pattern in cemented Oxford unicompartmental knee arthroplasty - an experimental cadaver study. Knee 2010, 17(6):398-402.

10. Price AJ, Dodd CA, Svard UG, Murray DW: Oxford medial unicompartmental knee arthroplasty in patients younger and older than 60 years of age. J Bone Joint Surg (Br) 2005, 87(11):1488-1492.

11. Langdown AJ, Pandit H, Price AJ, Dodd CA, Murray DW, Svard UC, Gibbons CL: Oxford medial unicompartmental arthroplasty for focal spontaneous osteonecrosis of the knee. Acta Orthop 2005, 76(5):688-692.

12. Luscombe KL, Lim J, Jones PW, White SH: Minimally invasive Oxford medial unicompartmental knee arthroplasty. A note of caution! Int Orthop 2007, 31(3):321-324.

13. Rea P, Short A, Pandit H, Price AJ, Kyberd P, Beard DJ, Gill HS, Murray DW: Radiolucency and migration after Oxford unicompartmental knee arthroplasty. Orthopedics 2007, 30(5 Suppl):24-27.

14. Pandit H, Murray DW, Dodd CA, Deo S, Waite J, Goodfellow J, Gibbons CL: Medial tibial plateau fracture and the Oxford unicompartmental knee. Orthopedics 2007, 30(5 Suppl):28-31.

15. Monk AP, Keys GW, Murray DW: Loosening of the femoral component after unicompartmental knee replacement. J Bone Joint Surg (Br) 2009, 91(3):405-407.

16. Choy WS, Kim KJ, Lee SK, Yang DS, Lee NK: Mid-term results of oxford medial unicompartmental knee arthroplasty. Clin Orthop Surg 2011, 3(3):178-183.

17. Meldrum RD, Wurtz LD, Feinberg JR, Capello WN: Does smoking affect implant survivorship in total hip arthroplasty? A preliminary retrospective case series. lowa Orthop J 2005, 25:17-24. 
18. Kim KT, Lee S, Cho KH, Kim KS: Fracture of the medial femoral condyle after unicompartmental knee arthroplasty. J Arthroplasty 2009, 24(7):1143.

19. Seeger JB, Jaeger S, Bitsch RG, Mohr G, Rohner E, Clarius M: The effect of bone lavage on femoral cement penetration and interface temperature during Oxford unicompartmental knee arthroplasty with cement. $J$ Bone Joint Surg Am 2013, 95(1):48-53.

doi:10.1186/1752-1947-8-419

Cite this article as: Argelo et al:: Femoral component failure in the Oxford unicompartmental knee arthroplasty: a case report. Journal of Medical Case Reports 2014 8:419.

\section{Submit your next manuscript to BioMed Central and take full advantage of:}

- Convenient online submission

- Thorough peer review

- No space constraints or color figure charges

- Immediate publication on acceptance

- Inclusion in PubMed, CAS, Scopus and Google Scholar

- Research which is freely available for redistribution 